九州大学学術情報リポジトリ

Kyushu University Institutional Repository

\title{
Realistic neutron energy spectrum and a possible enhancement of reaction rates in the early Universe plasma
}

Nakao, Yasuyuki

Department of Applied Quantum Physics and Nuclear Engineering, Kyushu University

Tsukida, Kazuki

Department of Applied Quantum Physics and Nuclear Engineering, Kyushu University

Voronchev, Victor T.

Institute of Nuclear Physics, Moscow State University

http://hdl. handle. net/2324/25711

出版情報: Physical Review D : Particles, Fields, Gravitation and Cosmology. 84 (6)， pp.063016(1)-063016(14), 2011-09-30. American Physical Society

バージョン:

権利関係: (C) 2011 American Physical Society 


\title{
Realistic neutron energy spectrum and a possible enhancement of reaction rates in the early Universe plasma
}

\author{
Yasuyuki Nakao, ${ }^{1}$ Kazuki Tsukida, ${ }^{1}$ and Victor T. Voronchev ${ }^{2}$ \\ ${ }^{1}$ Department of Applied Quantum Physics and Nuclear Engineering, Kyushu University, Motooka, Fukuoka 819-0395, Japan \\ ${ }^{2}$ Institute of Nuclear Physics, Moscow State University, Moscow 119991, Russia
}

(Received 21 June 2011; published 30 September 2011)

\begin{abstract}
A plasma-kinetic model to properly describe the behavior of neutrons in the primordial plasma during the epoch of big bang nucleosynthesis $(\mathrm{BBN})$ is formulated. For the first time, this model is applied to calculate the realistic energy distribution function of these neutrons and examine their main characteristics. The fact that the realistic neutron distribution departs from a conventional Maxwellian function is obtained; its high-energy tail is essentially enhanced by nonthermal neutrons produced in the $\mathrm{T}(d, n)^{4} \mathrm{He}$ and $\mathrm{D}(d, n)^{3} \mathrm{He}$ reactions. The fraction of these neutrons $\eta_{n}^{\prime}$ in the total neutron component is at a level of $10^{-2} \%-10^{-3} \%$, while their effective temperature $T_{n}^{\prime}$ reaches several $\mathrm{MeV}$ and exceeds the plasma temperature in the BBN epoch by about a factor of $10^{2}$. The nonthermal neutron influence on individual reactions is examined on the example of the threshold $\mathrm{D}(n, 2 n) p,{ }^{7} \mathrm{Li}(n, n t){ }^{4} \mathrm{He}$, and ${ }^{7} \mathrm{Be}\left(n, n^{3} \mathrm{He}\right)^{4} \mathrm{He}$ processes. We show that at plasma temperatures $T_{9} \lesssim 1.2$ the nonthermal neutrons strongly maintain these reactions, increasing their rates by orders of magnitude as compared with the respective Maxwellian estimates. Note that the obtained phenomenon has a general nature. It may manifest in other nuclear systems and becomes a natural supplement of nonthermal effects triggered by dark matter decay, which has been extensively studied elsewhere. An important question remains-to what extent such fast particles may affect chain reaction kinetics in the plasma and change the predictions of standard BBN.
\end{abstract}

DOI: 10.1103/PhysRevD.84.063016

PACS numbers: 26.35.+c, 95.30. $-\mathrm{k}$

\section{INTRODUCTION}

Big bang nucleosynthesis (BBN) still remains the most reliable probe of processes in the early Universe. These processes are commonly described in the standard model of BBN (SBBN), the basic points of which were formulated in $[1,2]$. A remarkable feature of this model is that SBBN initially operated with only one free parameter that was the baryon density $\Omega_{\mathrm{B}}$ (or the baryon-to-photon ratio $\eta=$ $n_{\gamma} / n_{\mathrm{B}}$ ) of the Universe. Furthermore, highly precise measurements of cosmic microwave background anisotropy by WMAP made an independent determination of $\Omega_{\mathrm{B}}$ possible $[3,4]$, and since that time SBBN has become a parameterfree theory. Probably, the most important consequence of this is that at present SBBN can serve as a unique tool to search for a signature of new physics, i.e., phenomena which cannot be understood in terms of conventional concepts. In this context we remind one that SBBN with the WMAP's baryon density gives reasonable predictions for the abundances of primordial $\mathrm{D}$ and ${ }^{4} \mathrm{He}$ in comparison with those inferred from observations, ${ }^{1}$ but essentially overestimates the amount of primordial ${ }^{7} \mathrm{Li}$ by a factor of 3-4.

In spite of many attempts to explain the ${ }^{7} \mathrm{Li}$ puzzle, this problem has not been resolved in SBBN yet and an updated picture does not look encouraging [6]. The phenomenon may in principle be considered as a candidate for new

\footnotetext{
${ }^{1}$ Note however a brief discussion in [5] that the currently adopted experimental abundance of primordial D may in principle be somewhat underestimated.
}

physics beyond the standard model that is widely discussed in the literature (see, e.g., [5,7-14], and references therein). To make it finally clear whether or not this fundamental cosmological conjecture is really valid, additional efforts are needed to further refine SBBN and improve the accuracy of its predictions. Such important work is currently in progress and, as an example, we note here a few issues to be focused on. These include an incorporation of some heretofore omitted processes [15-17] in the standard BBN reaction network $[18,19]$, a search for new resonant modes of reactions between light(est) nuclei [20,21], an inclusion of independent neutrino distribution functions in BBN modeling [22], an analysis of nuclear weak interaction rates [23], examinations of specific effects in the plasma related to thermally excited nuclei [17], electron screening [24], etc. The point of particular importance for refined BBN modeling is the use of updated rate parameters $\langle\sigma v\rangle$ of main nuclear reactions. These major nuclear inputs can be found in several comprehensive compilations [25-30]. These data were obtained using improved estimates of reaction cross sections and assuming the distribution functions of reacting particles to be Maxwellian. Thus, the standard BBN network operates with thermal reaction kinetics in the Maxwellian plasma.

There exists however an interesting issue relevant to suprathermal non-Maxwellian particles that are permanently present in the primordial plasma. These particles are generally produced due to two-primary and secondary-mechanisms. First of all, fast particles are straightforwardly generated in exoergic nuclear reactions 
and then remain in the plasma as suprathermal species until their thermalization is achieved. Besides, some fraction of bulk plasma particles can also be accelerated to suprathermal energies by recoil in close collisions between them and nonthermalized reaction products. As known, under certain conditions the fast products can transfer, in a single event, a large amount of energy. These two mechanisms increase the population of nonthermal particles, so the high-energy tails of particle distributions in the plasma can depart from a Maxwellian form. In other words, suprathermal particle groups appear in the plasma, and they trigger nonthermal reactions naturally supplementing thermal processes in the BBN epoch [31]. The rates of nonthermal reactions depend on the number densities $n^{\prime}$ of suprathermal particles and their effective temperatures $T^{\prime}$ being a measure of the particle average energy $\left\langle E^{\prime}\right\rangle$. The main characteristics of suprathermal particle groups were examined recently in [31]. That study demonstrated that slowing-down conditions for charged particles $c(=p, t$, ${ }^{3} \mathrm{He}, \alpha$ ) are not favorable to obtain an appreciable population of suprathermal $c$ groups. Coulomb interaction with a large amount of thermal electrons and positrons rapidly slows the fast charged particles, so their partial fractions $\eta_{c}=n_{c}^{\prime} / n_{c}$ in the respective $c$ components are negligibly small. At maximum $\eta_{c}$ ranges from $10^{-13}(c=p)$ to $10^{-6}$ $(c=t)$. Therefore, although the fast particle temperatures $T_{c}^{\prime}$ can be as high as $2 \mathrm{MeV}(c=p)$, the marginal amounts of these particles cannot provide a clear correction to the production of primordial $\mathrm{D},{ }^{3} \mathrm{He},{ }^{4} \mathrm{He}$, and ${ }^{7} \mathrm{Li}[31]$.

A more interesting situation concerns nonthermal neutrons. These neutral particles do not undergo the dramatic energy loss due to Coulomb scattering, and the neutron thermalization time $\tau_{n \text {,th }}$ proves to be larger by a few orders of magnitude than the thermalization times of charged particles $\tau_{c, \text { th }}$. This provides favorable conditions for the nonthermal neutron population to become comparatively high. It was clearly demonstrated that the most abundant nonthermal plasma species are neutrons, whose fraction $\eta_{n}^{\prime}=n_{n}^{\prime} / n_{n}$ in the total neutron component reaches $0.01 \%$ [31]. It is also interesting that at a Universe temperature of $T_{9} \simeq 0.9$ the amount of nonthermal neutrons even becomes comparable with the total amount of primordial ${ }^{3} \mathrm{He}$. Some remarkable properties of nonthermal neutronic reactions are also worth mentioning here. For example, at temperatures $T_{9}<0.8$ the $\mathrm{D}(n, 2 n)$ breakup induced by nonthermal neutrons is the key process that dominates over other possible reactions in the $N+\mathrm{D}$ system $(N=n, p)$ (a similar conclusion is also valid for reactions in the $N+{ }^{7} \mathrm{Li}$ system) [31]. Besides that, it was recently reported [32] that nonthermal neutrons can significantly change the relation between the forward $d+d \rightarrow$ $n+{ }^{3} \mathrm{He}$ and reverse $n+{ }^{3} \mathrm{He} \rightarrow d+d$ reactions in the plasma due to a strong enhancement of the reverse process. All this makes neutrons interesting objects for the analysis of nonthermal effects in SBBN.
A possible influence of nonthermal neutrons on chain reaction kinetics in the primordial plasma is determined by the fast neutron number density $n_{n}^{\prime}$ and effective temperature $T_{n}^{\prime}$. Some estimations of $n_{n}^{\prime}$ formed due to $\mathrm{D}+\mathrm{T}$ and $\mathrm{D}+\mathrm{D}$ reaction-produced neutrons are available in the literature $[16,17]$. However, the neutron temperature $T_{n}^{\prime}$ has still not been examined and remains an unknown parameter. In order to evaluate it accurately, first one needs to obtain a realistic neutron distribution function in the BBN epoch. A model approach [17] was recently applied to describe an energy distribution of fast neutrons in the plasma. The author considered DT fusion neutrons and examined their slowing down due to elastic scattering off bulk protons.

Several nuclear reactions can generate energetic neutrons in the BBN epoch [19]. The rates of such processes between nuclei $i$ and $j$ with mass number $A \leq 7$ (excluding rare ${ }^{6} \mathrm{Li}$ ) are shown in Fig. 1. These rates were defined in a conventional form as $R_{i j}=\left(1+\delta_{i j}\right)^{-1} n_{i} n_{j}\langle\sigma v\rangle_{i j}$ and obtained for the SBBN scenario with a WMAP's baryon-tophoton ratio $\eta=6.14 \times 10^{-10}$. The main objective of our work is to calculate the total neutron distribution function, having Maxwellian and non-Maxwellian components in the primordial plasma, within a realistic kinetic model and obtain its main characteristics. The model will consider two main neutron emitters (the $\mathrm{D}+\mathrm{T}$ and $\mathrm{D}+\mathrm{D}$ reactions) and allow for both downscattering $\left(n_{\text {fast }}+\right.$ slow particle $\rightarrow n_{\text {slow }}+$ fast particle) and upscattering $\left(n_{\text {bulk }}+\right.$ fast particle $\rightarrow n_{\text {fast }}+$ slow particle $)$ events to obtain the realistic distribution. This distribution then will be used to demonstrate the impact of nonthermal neutrons on some reaction rates on the example of $n$-induced two-body breakups of loosely bound $\mathrm{D},{ }^{7} \mathrm{Li}$, and ${ }^{7} \mathrm{Be}$. The paper is organized as follows. In Sec. II, mechanisms of fast neutron energy loss in the primordial plasma are analyzed. A plasma-kinetic model to properly describe the fast neutron component in the plasma during the BBN epoch is formulated in detail in Sec. III. In Sec. IV, we calculate the realistic neutron distribution function, examine its characteristics, and also discuss the influence of nonthermal neutrons on the rates of some individual reactions. The main conclusions of our study are summarized in Sec. V.

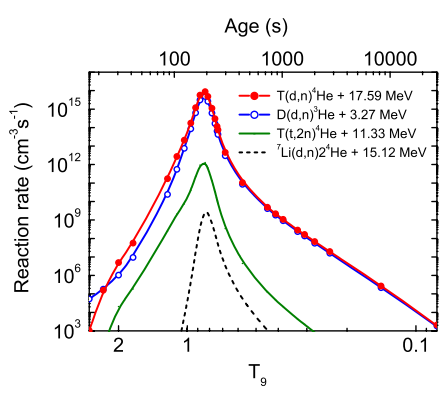

FIG. 1 (color online). The rates of reactions generating energetic neutrons as a function of the Universe temperature and age. 
TABLE I. The average rates of neutron energy losses $\left\langle d E_{n} / d t\right\rangle_{k}$ due to the NES, MMI, and MMC mechanisms.

\begin{tabular}{lcc}
\hline \hline Mechanism & Expression for $\left\langle d E_{n} / d t\right\rangle_{k}$ \\
\hline NES $^{\mathrm{a}}$ & $-2 \pi^{2} \sum_{j} \frac{m_{n} m_{j}}{m_{n}+m_{j}} n_{j} \int_{0}^{\infty} d v_{j} v_{j}^{2} \int_{-1}^{1} d \zeta u\left(v_{n}^{2}-v_{j}^{2}+\frac{m_{n}-m_{j}}{m_{n}+m_{j}} u^{2}\right) f_{j}\left(\mathbf{v}_{j}\right) \int_{-1}^{1} d \xi \sigma_{n j}(\xi, u)(1-\xi)$ & Ref. \\
MMI $^{\mathrm{b}}$ & $-\frac{16 \alpha_{n}^{2} g_{n}^{2}}{3 \pi\left(m_{n} c^{2}\right)^{3} \hbar} T^{4}\left(y_{e}^{3}+3 y_{e}^{2}+6 y_{e}+6\right) \exp \left(-y_{e}\right) E_{n}\left(\right.$ for $\left.m_{e} c^{2} / 26<T \leq m_{e} c^{2}\right)$ \\
& $\left.-\frac{4 \alpha^{2} g_{n}^{2} \hbar^{2} c^{3}}{3 \pi\left(m_{n} c^{2}\right)^{3}} n_{e}\left(\frac{2 \pi}{m_{e} c^{2} T^{3 / 2}}\right)^{3 / 2} m^{2}\right)^{3} T E_{n}\left(\right.$ for $\left.T \leq m_{e} c^{2} / 26\right)$ \\
MMC & $\frac{320}{441} \pi^{2} g_{n}^{2}\left(\frac{T}{m_{n} c^{2}}\right)^{2} \times\left\langle d E_{n} / d t\right\rangle_{\mathrm{MMI}}$
\end{tabular}

${ }^{\text {a }}$ The summation is taken over bulk nucleons and nuclei with number density $n_{j}$, mass $m_{j}$, and unit-normalized velocity distribution function $f_{j}\left(\mathbf{v}_{j}\right)$. The relative speed $u=\left|\mathbf{v}_{n}-\mathbf{v}_{j}\right|=\left(v_{n}^{2}+v_{j}^{2}-2 v_{n} v_{j} \zeta\right)^{1 / 2}$, where $\zeta$ is the cosine of angle between $\mathbf{v}_{n}$ and $\mathbf{v}_{j}$. The quantity $\sigma_{n j}(\xi, u)$ is the differential cross section of $n-j \mathrm{NES}$, where $\xi$ is the cosine of the scattering angle $\theta$ in the center-of-mass frame.

${ }^{\mathrm{b}}$ The quantity $y_{e}=m_{e} c^{2} / T, n_{e}\left(=n_{e^{-}}+n_{e^{+}}\right)$is the total number density of electrons and positrons, $\alpha(=1 / 137)$ is the fine structure constant, and $g_{n}(=-1.913)$ is the neutron magnetic moment.

\section{FAST NEUTRON ENERGY LOSS}

In this section, we consider mechanisms of energy loss of nonrelativistic neutrons in the primordial plasma and identify the most important of them. Main loss mechanisms will be incorporated in the kinetic model for fast neutron slowing down in the plasma described in the next section.

Nonrelativistic neutrons slow down in the plasma due to nuclear and electromagnetic scattering processes, such as

(1) nuclear elastic scattering (NES) off ambient nucleons $N$ and nuclei $\mathrm{A}(n+\mathrm{B} \rightarrow n+\mathrm{B})(\mathrm{B}=N, \mathrm{~A})$;

(2) magnetic-moment interaction (MMI) with background electrons $e^{-}$and positrons $e^{+}\left(n+e^{ \pm} \rightarrow\right.$ $\left.n+e^{ \pm}\right)$

(3) magnetic-moment coupling (MMC) to thermal photons $\gamma(n+\gamma \rightarrow n+\gamma)$.

Thus, the average energy loss rate of a fast neutron $\left\langle d E_{n} / d t\right\rangle$ is

$$
\left\langle\frac{d E_{n}}{d t}\right\rangle=\left\langle\frac{d E_{n}}{d t}\right\rangle_{\mathrm{NES}}+\left\langle\frac{d E_{n}}{d t}\right\rangle_{\mathrm{MMI}}+\left\langle\frac{d E_{n}}{d t}\right\rangle_{\mathrm{MMC}}
$$

where expressions for each loss term in Eq. (1) employed in our study are reduced in Table I.

The calculated energy loss rates are compared in Fig. 2. The solid curves show $\left\langle d E_{n} / d t\right\rangle_{\mathrm{NES}}$, while the dashed and dotted curves present the rates $\left\langle d E_{n} / d t\right\rangle_{\mathrm{MMI}}$ and $\left\langle d E_{n} / d t\right\rangle_{\mathrm{MMC}}$, respectively. These results are plotted for two different neutron energies $E_{n}=14.07 \mathrm{MeV}$ and 2.45 MeV typical of DT and DD fusion neutrons. It is seen that when $E_{n}$ drops, all the energy loss rates decrease but their comparative contribution to the total loss rate $\left\langle d E_{n} / d t\right\rangle$ does not change. At high temperatures, the total energy loss is governed by NES and MMI mechanisms. However, as the Universe cools, the number density of $e^{ \pm}$ pairs rapidly decreases and accordingly the MMI contribution drops. At temperatures $T_{9}<1$ of particular interest in our work, the main energy loss mechanism is NES.
It is worthwhile to identify which specific nuclear scattering process is most important here. Since the rate $\left\langle d E_{n} / d t\right\rangle_{\mathrm{NES}}$ is proportional to the number density of scattering centers $n_{j}$ (see Table I), the majority of energy loss through NES comes from the most abundant bulk particles $j$ in the plasma. These are protons, $\alpha$ particles at $T_{9} \lesssim 0.8$, and also neutrons at high temperatures $T_{9}>0.8$. The respective partial loss rates due to the $n$ - $p$ NES, $n-\alpha$ NES, and $n-n$ NES processes are plotted in Figs. 3 and 4. These results are found with $n-j$ scattering cross sections $d \sigma_{n j} / d \Omega$ taken from the ENDF [35]. Figure 3 shows temperature dependence of the partial loss rates on the
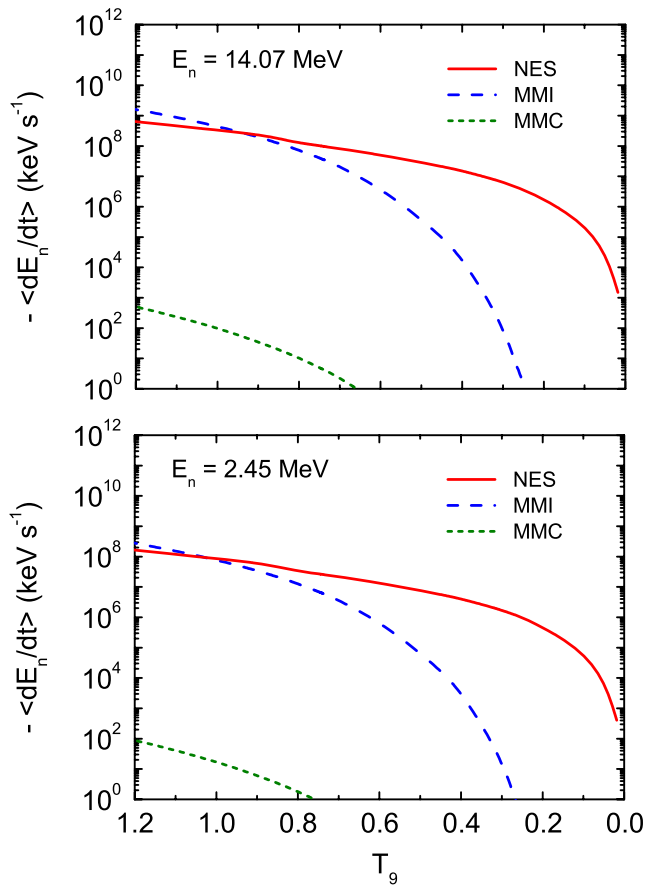

FIG. 2 (color online). The energy loss rates of fast neutrons due to the NES, MMI, and MMC mechanisms as a function of the Universe temperature. The upper and lower panels show the results for neutrons produced in the $\mathrm{D}+\mathrm{T}$ and $\mathrm{D}+\mathrm{D}$ reactions, respectively. 


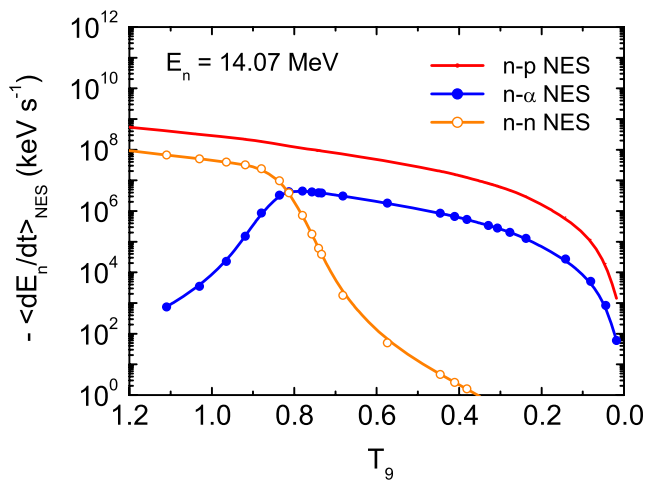

FIG. 3 (color online). The energy loss rates of DT neutrons due to NES off bulk plasma particles (protons, $\alpha$ particles, neutrons) as a function of the Universe temperature.



FIG. 4 (color online). The energy loss rates of fast neutrons due to NES off bulk plasma particles (protons, $\alpha$ particles, neutrons) as a function of neutron energy.

example of DT neutrons. Energy dependence of these rates at a moderate temperature $T_{9} \simeq 0.7$ is presented in Fig. 4 . A peak seen in the $n$ - $\alpha$ NES curve in Fig. 4 is accounted for by the $P_{3 / 2}$ resonance behavior of $n-\alpha$ scattering at $E_{n} \simeq 1 \mathrm{MeV}$ [36].

Summarizing the results, we can conclude that the dominant mechanism of fast neutron energy loss in the primordial plasma is NES off bulk protons. The next contribution to $\left\langle d E_{n} / d t\right\rangle_{\mathrm{NES}}$ at temperatures of our interest comes from elastic collisions of fast neutrons with bulk $\alpha$ particles.

\section{KINETIC MODEL DESCRIPTION}

In this section, we derive the kinetic equation for the slowing down and thermalization of fast neutrons generated in the primordial plasma. The nonrelativistic description of processes can be used because neutron energy $E_{n} \ll m_{n} c^{2}$ and plasma temperature in the BBN epoch $T<m_{e} c^{2}$.

\section{A. Basic equations}

We start from an equation of evolution for the velocity distribution function of fast neutrons $f_{n \text {,fast }}\left(\mathbf{v}_{n}, t\right)$

$$
\frac{\partial}{\partial t} f_{n, \text { fast }}\left(\mathbf{v}_{n}, t\right)=\left(\frac{\partial f_{n, \text { fast }}}{\partial t}\right)_{C}-L\left(\mathbf{v}_{n}, t\right)+S\left(\mathbf{v}_{n}, t\right),
$$

where $\left(\partial f_{n \text {,fast }} / \partial t\right)_{C}$ represents the scattering collision term, $L\left(\mathbf{v}_{n}, t\right)$ is the loss rate due to nuclear reactions, and $S\left(\mathbf{v}_{n}, t\right)$ is the source at an arbitrary time $t$.

Hereafter we omit for simplicity the subscript "fast," drop the time variable $t$, and write down the terms in Eq. (2). The scattering collision term consists of in- and outscattering rates, i.e., $\left(\partial f_{n} / \partial t\right)_{C}=\operatorname{In}\left(\mathbf{v}_{n}\right)-\operatorname{Out}\left(\mathbf{v}_{n}\right)$. Each rate is written in the Boltzmann integral form [37]

$$
\begin{aligned}
\operatorname{In}\left(\mathbf{v}_{n}\right)= & \sum_{j} \iiint f_{n}\left(\mathbf{v}_{n}^{\prime}\right) f_{j}\left(\mathbf{v}_{j}^{\prime}\right)\left|\mathbf{v}_{n}^{\prime}-\mathbf{v}_{j}^{\prime}\right| \\
& \times \sigma\left(\mathbf{v}_{n}^{\prime}, \mathbf{v}_{j}^{\prime} \rightarrow \mathbf{v}_{n}, \mathbf{v}_{j}\right) d \mathbf{v}_{n}^{\prime} d \mathbf{v}_{j}^{\prime} d \mathbf{v}_{j}, \\
\operatorname{Out}\left(\mathbf{v}_{n}\right)= & \sum_{j} \iiint f_{n}\left(\mathbf{v}_{n}\right) f_{j}\left(\mathbf{v}_{j}\right)\left|\mathbf{v}_{n}-\mathbf{v}_{j}\right| \\
& \times \sigma\left(\mathbf{v}_{n}, \mathbf{v}_{j} \rightarrow \mathbf{v}_{n}^{\prime}, \mathbf{v}_{j}^{\prime}\right) d \mathbf{v}_{n}^{\prime} d \mathbf{v}_{j} d \mathbf{v}_{j}^{\prime},
\end{aligned}
$$

where $f_{j}$ is the velocity distribution function of background ion species $j(j=p, \alpha, \ldots)$. According to the results of Sec. II, at temperatures of our interest $\left(T_{9}<1\right)$ the interaction between neutrons and those with plasma electrons as well as positrons can be neglected, i.e., $j \neq n, e^{-}, e^{+}$.

Let us introduce the probability distribution function $P$ which is defined for $\mathbf{v}_{n}$ and $\mathbf{v}_{n}^{\prime}$ (neutron velocities after scattering) so that $\int P\left(\mathbf{v}_{n}^{\prime} \rightarrow \mathbf{v}_{n}\right) d \mathbf{v}_{n}=\int P\left(\mathbf{v}_{n} \rightarrow\right.$ $\left.\mathbf{v}_{n}^{\prime}\right) d \mathbf{v}_{n}^{\prime}=1$. It follows that

$$
\begin{gathered}
\int \sigma\left(\mathbf{v}_{n}^{\prime}, \mathbf{v}_{j}^{\prime} \rightarrow \mathbf{v}_{n}, \mathbf{v}_{j}\right) d \mathbf{v}_{j}=\sigma_{S j}\left(u^{\prime}\right) P\left(\mathbf{v}_{n}^{\prime} \rightarrow \mathbf{v}_{n}\right), \\
\int \sigma\left(\mathbf{v}_{n}, \mathbf{v}_{j} \rightarrow \mathbf{v}_{n}^{\prime}, \mathbf{v}_{j}^{\prime}\right) d \mathbf{v}_{j}^{\prime}=\sigma_{S j}(u) P\left(\mathbf{v}_{n} \rightarrow \mathbf{v}_{n}^{\prime}\right),
\end{gathered}
$$

where $u^{\prime}=\left|\mathbf{v}_{n}^{\prime}-\mathbf{v}_{j}^{\prime}\right|, u=\left|\mathbf{v}_{n}-\mathbf{v}_{j}\right|$, and $\sigma_{S j}$ is the cross section of neutron scattering off background species $j$. Consequently, the scattering rates are reduced to

$$
\operatorname{In}\left(\mathbf{v}_{n}\right)=\sum_{j} \iint f_{n}\left(\mathbf{v}_{n}^{\prime}\right) f_{j}\left(\mathbf{v}_{j}^{\prime}\right) u^{\prime} \sigma_{S j}\left(u^{\prime}\right) P\left(\mathbf{v}_{n}^{\prime} \rightarrow \mathbf{v}_{n}\right) d \mathbf{v}_{n}^{\prime} d \mathbf{v}_{j}^{\prime}
$$

$$
\begin{aligned}
\operatorname{Out}\left(\mathbf{v}_{n}\right) & =\sum_{j} \iint f_{n}\left(\mathbf{v}_{n}\right) f_{j}\left(\mathbf{v}_{j}\right) u \sigma_{S j}(u) P\left(\mathbf{v}_{n} \rightarrow \mathbf{v}_{n}^{\prime}\right) d \mathbf{v}_{n}^{\prime} d \mathbf{v}_{j} \\
& =\sum_{j} \int f_{n}\left(\mathbf{v}_{n}\right) f_{j}\left(\mathbf{v}_{j}\right) u \sigma_{S j}(u) d \mathbf{v}_{j} .
\end{aligned}
$$

The fast neutrons disappear in the plasma through absorption reactions by the background species. The loss rate is hence written in terms of the absorption cross sections

$$
L\left(\mathbf{v}_{n}\right)=\sum_{j} \int f_{n}\left(\mathbf{v}_{n}\right) f_{j}\left(\mathbf{v}_{j}\right) u \sigma_{a j}(u) d \mathbf{v}_{j} .
$$


The main sources to generate fast neutrons in the plasma are fusion reactions and close scattering collisions between bulk thermal neutrons and some energetic particles. Accordingly, the generation rate may be written as

$$
S\left(\mathbf{v}_{n}\right)=S_{\text {fus }}\left(\mathbf{v}_{n}\right)+S_{\text {upscat }}\left(\mathbf{v}_{n}\right) .
$$

Now we put the equations into more tractable form. Because of the cosmological principle of homogeneity and isotropy of the Universe (in the standard model), one can assume that velocity distributions of neutrons and other plasma species are isotropic. We use the neutron energy $E_{n}$ instead of velocity $\mathbf{v}_{n}$ as an independent variable and adopt the neutron flux $\Psi\left(E_{n}\right)$ defined by $\Psi\left(E_{n}\right)=v_{n} f_{n}\left(E_{n}\right)$, where $f_{n}\left(E_{n}\right)$ is the neutron energy distribution function. The relation between $f_{n}\left(E_{n}\right)$ and the foregoing velocity distribution function is $f_{n}\left(\mathbf{v}_{n}\right) d \mathbf{v}_{n}=$ $f_{n}\left(v_{n}\right) 4 \pi v_{n}^{2} d v_{n}=f_{n}\left(E_{n}\right) d E_{n}$. The probability distribution function $P\left(E_{n}^{\prime} \rightarrow E_{n}\right)$ for $E_{n}$, scattered-neutron's energy, is also introduced. Its relation with the foregoing function is $P\left(\mathbf{v}_{n}^{\prime} \rightarrow \mathbf{v}_{n}\right) d \mathbf{v}_{n}=P\left(\mathbf{v}_{n}^{\prime} \rightarrow \mathbf{v}_{n}\right) 4 \pi v_{n}^{2} d v_{n}=$ $P\left(v_{n}^{\prime} \rightarrow v_{n}\right) d v_{n}=P\left(E_{n}^{\prime} \rightarrow E_{n}\right) d E_{n}$. Using these, first we rewrite Eq. (2) as

$$
\left(\frac{1}{v_{n}} \frac{\partial \Psi}{\partial t}\right)_{C}-L\left(E_{n}\right)+S\left(E_{n}\right)=0 .
$$

The components of the "new" scattering collision term $\left(v_{n}^{-1} \partial \Psi / \partial t\right)_{C}$ are treated as follows:

$$
\begin{aligned}
\operatorname{In}\left(E_{n}\right)= & \sum_{j} \iint f_{n}\left(E_{n}^{\prime}\right) f_{j}\left(E_{j}\right) u^{\prime} \sigma_{S j}\left(u^{\prime}\right) P\left(E_{n}^{\prime} \rightarrow E_{n}\right) d E_{n}^{\prime} d E_{j} \\
= & \sum_{j} \int \Psi\left(E_{n}^{\prime}\right)\left[\frac{1}{v_{n}^{\prime}} \int u^{\prime} \sigma_{S j}\left(u^{\prime}\right)\right. \\
& \left.\times P\left(E_{n}^{\prime} \rightarrow E_{n}\right) f_{j}\left(E_{j}\right) d E_{j}\right] d E_{n}^{\prime} \\
= & \sum_{j} \int n_{j} \sigma_{S j}^{\mathrm{eff}}\left(E_{n}^{\prime} \rightarrow E_{n}\right) \Psi\left(E_{n}^{\prime}\right) d E_{n}^{\prime},
\end{aligned}
$$

where $n_{j}$ is the number density of background species $j$, and $\sigma_{S j}^{\text {eff }}\left(E_{n}^{\prime} \rightarrow E_{n}\right)$ is the differential scattering cross section averaged over the energy distribution of background species $j$, i.e.,

$\sigma_{S j}^{\mathrm{eff}}\left(E_{n}^{\prime} \rightarrow E_{n}\right) \equiv \frac{1}{n_{j} v_{n}^{\prime}} \int u^{\prime} \sigma_{S j}\left(u^{\prime}\right) P\left(E_{n}^{\prime} \rightarrow E_{n}\right) f_{j}\left(E_{j}\right) d E_{j}$.

Similarly, the outscattering rate is modified as

$$
\begin{aligned}
\operatorname{Out}\left(E_{n}\right) & =\sum_{j} \iint f_{n}\left(E_{n}\right) f_{j}\left(E_{j}\right) u \sigma_{S j}(u) P\left(E_{n} \rightarrow E_{n}^{\prime}\right) d E_{n}^{\prime} d E_{j} \\
& =\sum_{j} \Psi\left(E_{n}\right) v_{n}^{-1} \int u \sigma_{S j}(u) f_{j}\left(E_{j}\right) d E_{j} \\
& =\sum_{j} n_{j} \sigma_{S j}^{e f f}\left(E_{n}\right) \Psi\left(E_{n}\right)
\end{aligned}
$$

where $\sigma_{S j}^{\text {eff }}\left(E_{n}\right)$ is the scattering cross section averaged over the thermal motion of target $j$

$$
\sigma_{S j}^{\mathrm{eff}}\left(E_{n}\right) \equiv \frac{1}{n_{j} v_{n}} \int u \sigma_{S j}(u) f_{j}\left(E_{j}\right) d E_{j}
$$

The loss rate is given by

$$
\begin{aligned}
L\left(E_{n}\right) & =\sum_{j} \int f_{n}\left(E_{n}\right) f_{j}\left(E_{j}\right) u \sigma_{a j}(u) d E_{j} \\
& =\sum_{j} \Psi\left(E_{n}\right) v_{n}^{-1} \int u \sigma_{a j}(u) f_{j}\left(E_{j}\right) d E_{j} \\
& =\sum_{j} n_{j} \sigma_{a j}^{\mathrm{eff}}\left(E_{n}\right) \Psi\left(E_{n}\right)
\end{aligned}
$$

where $\sigma_{a j}^{\mathrm{eff}}\left(E_{n}\right)$ is the thermal-motion average of the reaction cross section.

Combining these terms and using the total cross section $\sigma_{t j}^{\text {eff }}=\sigma_{S j}^{\text {eff }}+\sigma_{a j}^{\text {eff }}$, we obtain the final form of the kinetic equation for fast neutron slowing down and thermalization

$$
\begin{aligned}
& \frac{1}{v_{n}} \frac{\partial \Psi}{\partial t}+\sum_{j} n_{j}(t) \sigma_{t j}^{\mathrm{eff}}\left(E_{n}\right) \Psi\left(E_{n}, t\right) \\
& =\sum_{j} \int n_{j}(t) \sigma_{S j}^{\mathrm{eff}}\left(E_{n}^{\prime} \rightarrow E_{n}\right) \Psi\left(E_{n}^{\prime}, t\right) d E_{n}^{\prime}+S\left(E_{n}, t\right) .
\end{aligned}
$$

In Eq. (17) we have recovered the time variable $t$. However, in most cases, the neutron thermalization time $\tau_{n \text {,th }}$ is much shorter than a typical time scale of plasma evolution in the BBN epoch (see, e.g., [16]). This implies that the plasma temperature and density can be assumed to "freeze out" during the time needed for the slowing down and thermalization of fast neutrons. In such a case, we can make "steady-state" calculations to obtain the neutron velocity distribution function at each time step $t_{n}$. The equation to be solved is hence

$$
\begin{aligned}
& \sum_{j} n_{j} \sigma_{t j}^{\mathrm{eff}}\left(E_{n}\right) \Psi\left(E_{n}\right) \\
& \quad=\sum_{j} \int n_{j} \sigma_{S j}^{\mathrm{eff}}\left(E_{n}^{\prime} \rightarrow E_{n}\right) \Psi\left(E_{n}^{\prime}\right) d E_{n}^{\prime}+S\left(E_{n}\right) .
\end{aligned}
$$

From this equation we can derive the energy distribution of fast neutrons at each time step in the BBN epoch. For accurate calculations, thermal-motion averaging of the cross sections is essential.

\section{B. Fast neutron source}

The source strength $S\left(E_{n}\right)$ consists of fusion and upscattering components. The rate due to a fusion reaction $i+j \rightarrow k+n$, for example, is given by [38] 


$$
\begin{aligned}
S_{j(i, n) k}\left(E_{n}\right) & =\frac{1}{1+\delta_{i j}} n_{i} n_{j}\langle\sigma v\rangle_{i j} \frac{1}{\sqrt{\pi} \Delta} \exp \left[-\frac{\left(E_{n}-E_{0}\right)^{2}}{\Delta^{2}}\right], \\
\Delta & =\sqrt{\frac{4 m_{n} T E_{0}}{m_{k}+m_{n}}}, \quad E_{0}=\frac{m_{k}}{m_{k}+m_{n}} Q
\end{aligned}
$$

where $Q$ is the reaction $Q$ value, and $T$ is the plasma temperature in energy units.

The upscattering rate of bulk thermal neutrons due to close collisions of energetic particles (e.g., fast protons released in the $\mathrm{D}+{ }^{3} \mathrm{He}$ fusion reaction) is written in terms of their flux $\Psi_{k}$ and appropriate differential scattering cross sections as

$$
S_{\text {upscat }}\left(E_{n}\right)=\sum_{k} \int n_{n, \text { bulk }} \sigma_{S n}^{\text {eff }}\left(E_{k}^{\prime} ; E_{n}\right) \Psi_{k}\left(E_{k}^{\prime}\right) d E_{k}^{\prime} .
$$

Here, we have defined $\sigma_{S n}^{\text {eff }}\left(E_{k}^{\prime} ; E_{n}\right)$, the effective differential cross section for the energetic particle $k$ with energy $E_{k}^{\prime}$ to undergo a close scattering collision such that a thermal neutron is knocked into a unit energy width around $E_{n}$. If the scattering is elastic, from energy conservation $\sigma_{S n}^{\text {eff }}\left(E_{k}^{\prime} ; E_{n}\right)=\sigma_{S n}^{\text {eff }}\left(E_{k}^{\prime} \rightarrow E_{k}^{\prime}+E-3 T / 2\right)$.

To calculate $S_{\text {upscat }}\left(E_{n}\right)$ we need $\Psi_{k}\left(E_{k}^{\prime}\right)$, the flux of energetic charged particles. An appropriate kinetic equation to obtain $\Psi_{k}\left(E_{k}^{\prime}\right)$ is given, for example, in our previous paper [39]. There, the equation is being written for velocity distribution function $f_{k}\left(v_{k}\right)$. The scattering collision term consists of the Fokker-Planck term treating small-angle Coulomb scattering and the Boltzmann integral for close collisions. An additional term is also included in the equation to allow for the particle loss from the "confinement system." In this study, we omit this additional term and use the equation to calculate $f_{k}\left(\boldsymbol{v}_{k}\right)$.

\section{Thermal-motion averaging of cross sections}

The averaging procedure is somewhat complicated because the integrands in Eqs. (13) and (15) include relative speeds $u^{\prime}$ and $u$, respectively. To advance the integration operations, we return for a moment to the velocity representation.

The averaging technique is found in the literature on nuclear reactor theory [40] or neutron physics [41]. The basic formula for thermal-motion averaging of cross sections is

$$
\sigma_{S j}^{\mathrm{eff}}\left(v_{n}\right)=\frac{1}{n_{j} v_{n}} \int u \sigma_{S j}(u) f_{j}\left(\mathbf{v}_{j}\right) d \mathbf{v}_{j}
$$

In evaluating, most of the texts neglect the energy dependence of the cross section; the energy-independent cross section $\sigma_{S 0 j}$ for a target at rest is used instead of $\sigma_{S j}(u)$. In our case, however, such a treatment is not always valid, so the cross section (scattering, absorption) is operated as

$$
\begin{aligned}
\sigma_{S j}^{\mathrm{eff}}\left(v_{n}\right) & =\frac{1}{n_{j} v_{n}} \int u \sigma_{S j}(u) f_{j}\left(\mathbf{v}_{j}\right) d \mathbf{v}_{j} \\
& =\frac{1}{n_{j} v_{n}} 2 \pi \int_{0}^{\infty} \int_{0}^{\pi} u \sigma_{S j}(u) f_{j}\left(\mathbf{v}_{j}\right) v_{j}^{2} \sin \theta d v_{j} d \theta,
\end{aligned}
$$

where $\theta$ is the angle between $\mathbf{v}_{n}$ and $\mathbf{v}_{j}$. From the relation $u^{2}=v_{n}^{2}+v_{j}^{2}-2 v_{n} v_{j} \cos \theta, \quad \sin \theta d \theta=u d u / v_{n} v_{j}$. The interval that corresponds to $0 \leq \theta \leq \pi$ is $\left|v_{n}-v_{j}\right| \leq u \leq$ $v_{n}+v_{j}$. Thus, we obtain

$$
\begin{aligned}
\sigma_{S j}^{\mathrm{eff}}\left(v_{n}\right)= & \frac{1}{n_{j} v_{n}^{2}} 2 \pi \int_{0}^{\infty} v_{j} f_{j}\left(v_{j}\right) \\
& \times\left[\int_{\left|v_{n}-v_{j}\right|}^{v_{n}+v_{j}} u^{2} \sigma_{S j}(u) d u\right] d v_{j} .
\end{aligned}
$$

The energy-variable representation of Eq. (23) is given by

$$
\begin{aligned}
\sigma_{S j}^{\mathrm{eff}}\left(E_{n}\right)= & \frac{m_{n} \sqrt{m_{j}}}{4 m_{r}^{3 / 2} n_{j} E_{n}} \int_{0}^{\infty} E_{j}^{-1 / 2} f_{j}\left(E_{j}\right) \\
& \times\left[\int_{E_{r}^{-}}^{E_{r}^{+}} E_{r}^{1 / 2} \sigma_{S j}\left(E_{r}\right) d E_{r}\right] d E_{j},
\end{aligned}
$$

where $m_{r}$ is the reduced mass, and $E_{r} \equiv m_{r} u^{2} / 2$ is the relative energy. We defined $E_{r}^{+(-)}$by

$$
E_{r}^{+(-)} \equiv \frac{1}{2} m_{r}\left[\sqrt{\frac{2 E_{n}}{m_{n}}}+(-) \sqrt{\frac{2 E_{j}}{m_{j}}}\right]^{2} .
$$

In a similar way, the thermal-motion averaging of the differential scattering cross section is written as

$$
\begin{aligned}
\sigma_{S j}^{\mathrm{eff}}\left(v_{n}^{\prime} \rightarrow v_{n}\right)= & \frac{1}{n_{j} v_{n}^{\prime}} \int u^{\prime} \sigma_{S j}\left(u^{\prime}\right) P\left(v_{n}^{\prime} \rightarrow v_{n}\right) f_{j}\left(\mathbf{v}_{j}\right) d \mathbf{v}_{j} \\
= & \frac{1}{n_{j} v_{n}^{\prime 2}} 2 \pi \int_{0}^{\infty} v_{j} f_{j}\left(v_{j}\right)\left[\int_{\left|v_{n}^{\prime}-v_{j}\right|}^{v_{n}^{\prime}+v_{j}} u^{\prime 2} \sigma_{S j}\left(u^{\prime}\right)\right. \\
& \left.\times P\left(v_{n}^{\prime} \rightarrow v_{n}\right) d u^{\prime}\right] d v_{j} .
\end{aligned}
$$

When the scattering is elastic, the probability distribution function $P$ is given by [40]

$$
P\left(v_{n}^{\prime} \rightarrow v_{n}\right)=\frac{4 v_{n} g\left(v_{n}^{\prime}, \mu_{C}\right)}{v_{n, \max }^{2}-v_{n, \min }^{2}},
$$

where $g\left(v_{n}^{\prime}, \mu_{C}\right)$ is the probability distribution function for the cosine of the scattering angle in the center-of-mass system. If the scattering is isotropic, then $g\left(v_{n}^{\prime}, \mu_{C}\right)=$ $1 / 2$. The upper and lower limits of the possible "final" speed $v_{n}$ are given by

$$
v_{n, \max (\min )}^{2}=V_{\mathrm{G}}+\frac{m_{r}^{2} u^{2}}{\left(m_{n}+m_{j}\right)^{2}}+(-) \frac{2 m_{j} V_{\mathrm{G}} u^{\prime}}{m_{n}+m_{j}},
$$

where $V_{\mathrm{G}}$ is the speed of the center of mass 


$$
\begin{aligned}
V_{\mathrm{G}} & =\left|\frac{m_{n} \mathbf{v}_{n}^{\prime}+m_{j} \mathbf{v}_{j}}{m_{n}+m_{j}}\right| \\
& =\frac{\sqrt{\left(m_{n}+m_{j}\right)\left(m_{n} v_{n}^{\prime 2}+m_{j} v_{j}^{2}\right)-m_{n} m_{j} u^{\prime 2}}}{m_{n}+m_{j}} .
\end{aligned}
$$

The energy-variable representation of Eq. (26) is

$$
\begin{aligned}
\sigma_{S j}^{\mathrm{eff}}\left(E_{n}^{\prime} \rightarrow E_{n}\right)= & \frac{m_{n} \sqrt{m_{j}}}{4 m_{r}^{3 / 2} n_{j} E_{n}^{\prime}} \int_{0}^{\infty} E_{j}^{-1 / 2} f_{j}\left(E_{j}\right) \\
& \times\left[\int_{E_{r}^{\prime-}}^{E_{r}^{\prime+}} E_{r}^{\prime 1 / 2} \sigma_{S j}\left(E_{r}^{\prime}\right) P\left(E_{n}^{\prime} \rightarrow E_{n}\right) d E_{r}^{\prime}\right] d E_{j} .
\end{aligned}
$$

The upper and lower limits of the inner integral are

$$
E_{r}^{\prime+(-)} \equiv \frac{1}{2} m_{r}\left[\sqrt{\frac{2 E_{n}^{\prime}}{m_{n}}}+(-) \sqrt{\frac{2 E_{j}}{m_{j}}}\right]^{2} .
$$

The probability distribution function $P$ is written as

$$
P\left(E_{n}^{\prime} \rightarrow E_{n}\right)=\frac{2 g\left(E_{n}^{\prime}, \mu_{C}\right)}{E_{n, \max }-E_{n, \min }},
$$

where $E_{n, \max (\min )}$ is the upper (lower) limit of possible final energy $E_{n}$ :

$$
\begin{aligned}
E_{n, \max (\min )}= & \frac{1}{2} m_{n} v_{n, \max (\min )}^{2} \\
= & \frac{m_{n}\left(E_{n}^{\prime}+E_{n}\right)}{m_{n}+m_{j}}-\frac{\left(m_{n}-m_{j}\right) E_{r}^{\prime}}{m_{n}+m_{j}}+(-) \\
& \times \frac{2 \sqrt{m_{n} m_{j}\left(E_{n}^{\prime}+E_{j}-E_{r}^{\prime}\right) E_{r}^{\prime}}}{m_{n}+m_{j}} .
\end{aligned}
$$

\section{Numerical method}

Equation (18) is solved numerically. There is no new feature in the numerical method itself, so we here describe only the outline of the method adopted, i.e., the "multigroup method" [40]. First, the neutron energy range of interest is divided into a finite number $N$ of intervals separated by the energies $E_{g}$, i.e. $E_{N}<$ $E_{N-1}<\cdots<E_{g}<E_{g-1}<\cdots<E_{1}<E_{0}$. Then, the group-integrated flux and source are defined by

$$
\Psi_{g} \equiv \int_{E_{g}}^{E_{g-1}} \Psi\left(E_{n}\right) d E_{n}, \quad S_{g} \equiv \int_{E_{g}}^{E_{g-1}} S\left(E_{n}\right) d E_{n} .
$$

Integrating the energy-dependent equation (18) over the $g$ th energy interval $\left[E_{g}, E_{g-1}\right]$, we obtain the multigroup equation,

$$
\sum_{j} n_{j} \sigma_{t j, g}^{\mathrm{eff}} \Psi_{g}=\sum_{j} \sum_{h} n_{j} \sigma_{S j, h \rightarrow g}^{\mathrm{eff}} \Psi_{h}+S_{g},
$$

where $\sigma_{t j, g}^{\text {eff }}$ and $\sigma_{S j, h \rightarrow g}^{\text {eff }}$ are "group cross sections" or "group constants" defined, respectively, by

$$
\begin{gathered}
\sigma_{t j, g}^{\mathrm{eff}} \equiv \frac{\int_{E_{g}}^{E_{g-1}} \sigma_{t j}^{\mathrm{eff}}\left(E_{n}\right) \Psi\left(E_{n}\right) d E_{n}}{\int_{E_{g}}^{E_{g-1}} \Psi\left(E_{n}\right) d E_{n}} \\
=\frac{1}{\Psi_{g}} \int_{E_{g}}^{E_{g-1}} \sigma_{t j}^{\mathrm{eff}}\left(E_{n}\right) \Psi\left(E_{n}\right) d E_{n} \\
\sigma_{t j, h \rightarrow g}^{\mathrm{eff}} \equiv \frac{\int_{E_{g}}^{E_{g-1}} \int_{E_{h}}^{E_{h-1}} \sigma_{t j}^{e f f}\left(E_{n}^{\prime} \rightarrow E_{n}\right) \Psi\left(E_{n}^{\prime}\right) d E_{n}^{\prime} d E_{n}}{\int_{E_{h}}^{E_{h-1}} \Psi\left(E_{n}^{\prime}\right) d E_{n}^{\prime}} \\
=\frac{1}{\Psi_{g}} \int_{E_{g}}^{E_{g-1}} \int_{E_{h}}^{E_{h-1}} \sigma_{t j}^{e f f}\left(E_{n}^{\prime} \rightarrow E_{n}\right) \Psi\left(E_{n}^{\prime}\right) d E_{n}^{\prime} d E_{n} .
\end{gathered}
$$

Equation (35) is solved by iteration to obtain the group flux $\Psi_{g}(g=1,2, \ldots, N)$. From $\Psi_{g}$ we construct the distribution function of fast neutrons- $-f_{n \text {,fast }}\left(E_{n}\right)$.

\section{NUMERICAL RESULTS AND DISCUSSIONS}

In this section we calculate the realistic distribution function of plasma neutrons in the BBN epoch and examine its main characteristics. We also demonstrate how the non-Maxwellian perturbation of this distribution can change the rates of some neutron-induced reactions.

\section{A. Neutron distribution function}

In order to solve Eq. (18) one needs to specify processes most important for scattering, absorption, and generation of fast neutrons in the plasma. Having in mind the results of Sec. II, in the present calculations we allow for the neutron interaction with bulk protons and also $\alpha$ particles. The respective $n-p$ and $n$ - $\alpha$ total (or elastic) cross sections, $\sigma_{t j}\left(\right.$ or $\sigma_{S j}$ ), are taken from [35]. The fast neutron source $S$ in Eq. (18) is a sum of fusion and upscattering terms. The fusion term $S_{\text {fus }}$ is mainly determined by the $\mathrm{T}(d, n)^{4} \mathrm{He}$ and $\mathrm{D}(d, n)^{3} \mathrm{He}$ reactions which are dominant neutron emitters in the BBN epoch (see Fig. 1). Energetic particles - the most appropriate candidates for contribution in the upscattering term $S_{\text {upscat }}$-are $14.68-\mathrm{MeV}$ protons released in the ${ }^{3} \mathrm{He}(d, p){ }^{4} \mathrm{He}$ fusion reaction. Indeed, this reaction is an effective emitter of highly energetic protons and, besides, the kinematics of $n-p$ elastic collisions provides favorable conditions for energy transfer from $p$ (fast) to $n$ (slow).

Figure 5 presents these source strengths at plasma temperatures $T_{9}=0.9$ and 0.1 . The solid curves show $S_{\text {fus }}\left(E_{n}\right)$ for DT and DD neutrons. The shape of $S_{\text {fus }}$ has a Gaussian form with temperature-dependent width given by Eq. (19). Note that the shape asymmetry, especially seen at $T_{9}=$ 0.9 , simply results from a logarithmic scale of energy axis. The dashed curves give $S_{\text {upscat }}\left(E_{n}\right)$, the knock-on neutron source due to collisions between bulk neutrons and fast 
$\mathrm{D}^{3} \mathrm{He}$ protons. These curves are truncated at an energy point corresponding to the maximum kinetic energy gained by a bulk neutron in head-on backscattering by the 14.68$\mathrm{MeV}$ proton. Thus, Fig. 5 shows that the main sources of neutrons with energy $E_{n}$ in excess of $1 \mathrm{MeV}$ are the D + T and $\mathrm{D}+\mathrm{D}$ reactions, while the $n-p$ upscattering process essentially contributes to the production of neutrons with $E_{n}<1 \mathrm{MeV}$.

Now we consider the neutron energy distribution. The realistic (total) distribution function $f_{n}\left(E_{n}\right)$ of neutrons at a cosmic time $t$ can reasonably be defined as

$$
f_{n}\left(E_{n}\right)=f_{n, \text { fast }}\left(E_{n}\right)+f_{n, \text { bulk }}\left(E_{n}\right),
$$

where $f_{n \text {,fast }}$ is the fast neutron distribution introduced in Sec. III, while $f_{n \text {,bulk }}$ is the thermal distribution of bulk neutrons populating the primordial plasma at that time $t$. Figure 6 presents, as an example, neutron energy distributions calculated at three different temperatures $T_{9}=0.9$, 0.7 , and 0.1 . The solid curves show the realistic distribution functions allowing for the fast neutron component. For comparison, the respective Maxwellian distributions are indicated by the dashed curves. It is clearly seen that the high-energy tail of the realistic distribution is appreciably enhanced and deviates from the Maxwellian function. This phenomenon is a result of the plasma irradiation (pumping) by fast 14.07-MeV DT and 2.45-MeV DD fusion neutrons. ${ }^{2}$ Note that one can discern two peaks in the realistic distribution tail located at energies corresponding to the birth energies of these reaction-produced particles. Figure 6 indicates that the non-Maxwellian perturbation of the neutron distribution function becomes more pronounced as the Universe expands and cools.

It is also useful to plot a function $y_{n}\left(E_{n}\right)=E_{n} f_{n}\left(E_{n}\right)$. This function is easily reduced to $y_{n}\left(E_{n}\right)=d n_{n} / d\left(\ln E_{n}\right)$ and thus gives the "spectral" abundance of neutrons, i.e., the neutron abundance per log energy interval. As an example, Fig. 7 shows $y_{n}\left(E_{n}\right)$ for $T_{9}=0.7$.

\section{B. Nonthermal neutron parameters}

In order to describe the obtained perturbation quantitatively, one needs to evaluate the number density $n_{n}^{\prime}$ and the effective temperature $T_{n}^{\prime}$ of nonthermal neutrons populating the non-Maxwellian distribution function tail.

\footnotetext{
${ }^{2}$ We briefly comment on the form of the neutron distribution tail. It is well known that when an infinite, homogeneous, hydrogenous medium contains sources emitting neutrons with the energy $E_{0}$ at a constant rate and when there is no absorption, the flux of moderated neutrons follows a $1 / E_{n}$ law [41]. Thus, in our case at energies below $E_{0}$ the neutron distribution function $f_{n}\left(E_{n}\right)$ becomes approximately proportional to $E_{n}^{-3 / 2}$. A nearly linear form of nonthermal spectra seen in Fig. 6 (double log scale) reflects this fact.
}

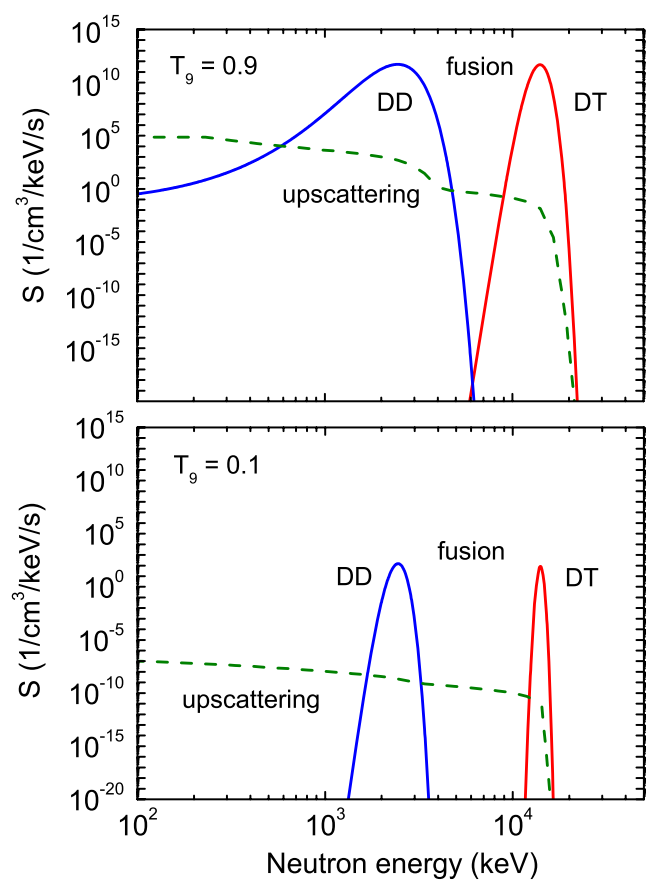

FIG. 5 (color online). The source strength $S\left(E_{n}\right)$ of fast neutrons in the plasma at temperatures $T_{9}=0.9$ and 0.1 . Shown are the fusion and upscattering components, $S_{\text {fus }}$ and $S_{\text {upscat }}$ (see details in the text).

The value of $n_{n}^{\prime}$ is given by

$$
n_{n}^{\prime}=\int_{E_{1}}^{E_{2}} f_{n}^{\text {tail }}\left(E_{n}\right) d E_{n},
$$

where $f_{n}^{\text {tail }}$ is the non-Maxwellian tail of the realistic neutron distribution and the integration limits $E_{1}$ and $E_{2}$ can easily be determined using Fig. 6. The fraction of nonthermal neutrons in the total neutron component is $\eta_{n}^{\prime}=n_{n}^{\prime} / n_{n}$. The dilution of $n_{n}^{\prime}$ due to expansion can be factored out by using the reduced number density of nonthermal neutrons defined as $n_{n}^{\prime} / n_{\mathrm{B}}$, where $n_{\mathrm{B}}$ is the baryon number density. The quantity $n_{n}^{\prime} / n_{\mathrm{B}}$ is shown in Fig. 8. We see that at $T_{9} \simeq 0.8$ the nonthermal neutrons account for $2 \times 10^{-7}$ of the total number of bound and free nucleons $N(=n, p)$ in the Universe. The fraction of nonthermal neutrons $\eta_{n}^{\prime}$ in percent is presented in Fig. 9. The solid curve shows $\eta_{n}^{\prime}$ calculated in the present work. It has a steplike form and its "plateau" ranges within $10^{-2} \%-10^{-3} \%$. The dotted curve gives $\eta_{n}^{\prime}$ found by an independent method, where the number density of nonthermal neutrons produced in the $\mathrm{D}+\mathrm{T}$ and $\mathrm{D}+\mathrm{D}$ reactions is estimated as

$$
n_{n}^{\prime}=R_{\mathrm{DT}} \times \tau_{n, \mathrm{th}}+R_{\mathrm{DD}} \times \tau_{n, \mathrm{th}} .
$$

Here $R_{i j}$ is the $i+j$ reaction rate [see Eq. (43) in the next section] and $\tau_{n, \text { th }}$ is the neutron thermalization time defined by 

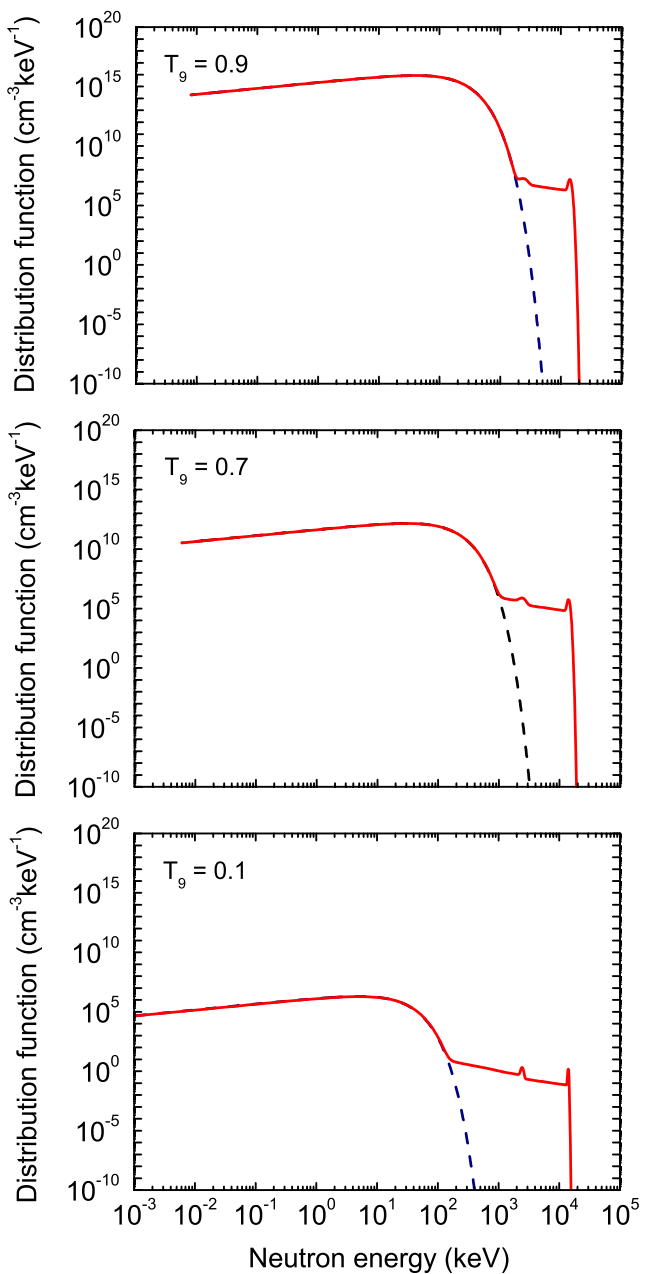

FIG. 6 (color online). Neutron distribution functions in the primordial plasma at three different temperatures $T_{9}=0.9$, 0.7 , and 0.1 . The solid curves show the calculated realistic distribution functions. The respective Maxwellian distributions are given by the dashed curves.



FIG. 7 (color online). The neutron abundance per log energy interval as a function of neutron energy.

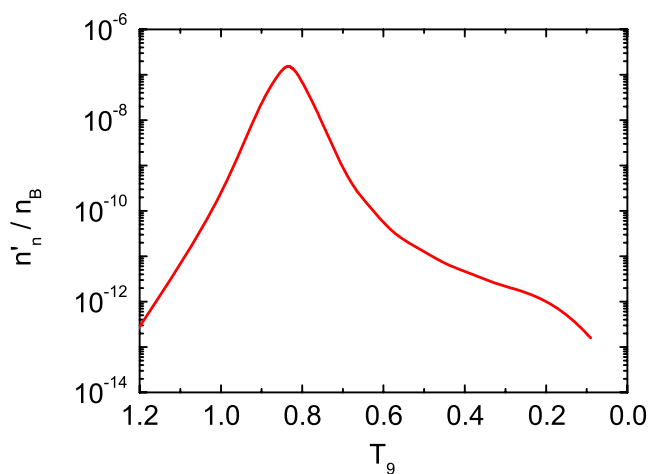

FIG. 8 (color online). The reduced number density of nonthermal neutrons $n_{n}^{\prime} / n_{\mathrm{B}}$ as a function of the Universe temperature.

$$
\tau_{n, \mathrm{th}}=\int_{E_{\mathrm{th}}}^{E_{0}}-d E_{n} /\left\langle d E_{n} / d t\right\rangle
$$

where $E_{0}$ is the neutron birth energy in the respective reaction, and $E_{\mathrm{th}}=3 T / 2$ is the plasma thermal energy. It is seen that this method somewhat overestimates the nonthermal neutron fraction but reasonably reproduces its dependence on plasma temperature. Note that a similar steplike behavior of $\eta_{n}^{\prime}\left(T_{9}\right)$ was obtained in a kinetic analysis [17].

For comparison, we also demonstrate in Fig. 9 the respective result for charged particles on the example of nonthermal $\mathrm{D}^{3} \mathrm{He}$ protons. Their number density was estimated as $n_{p}^{\prime}=R_{\mathrm{D}^{3} \mathrm{He}} \times \tau_{p \text {,th }}$. The fraction of these protons $\eta_{p}^{\prime}$ in primordial hydrogen is shown by the dashed curve. Rapid thermalization of the $\mathrm{D}^{3} \mathrm{He}$ protons through electromagnetic interaction with plasma species (mainly with $e^{ \pm}$) strongly suppresses $n_{p}^{\prime}$ and makes $\eta_{p}^{\prime} \ll \eta_{n}^{\prime}$. The negligible nonthermal proton abundance is a reason why the $n-p$ upscattering process does not play an essential role in the production of high-energy neutrons (see Fig. 5).

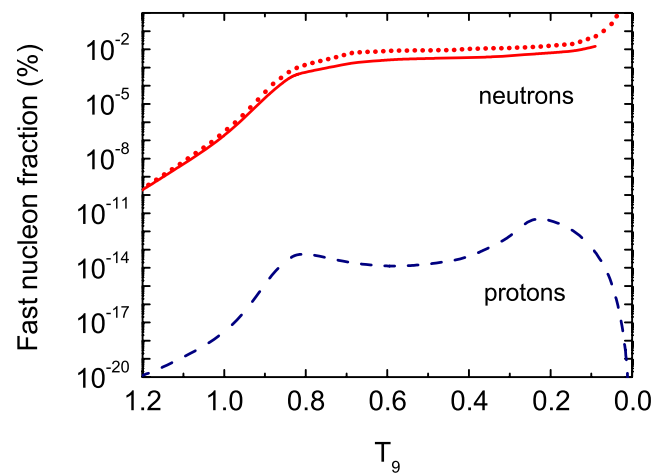

FIG. 9 (color online). The fraction of nonthermal neutrons $\eta_{n}^{\prime}=n_{n}^{\prime} / n_{n}$ as a function of the Universe temperature. The realistic and model estimates of $\eta_{n}^{\prime}$ are shown by the solid and dotted curves, respectively. For comparison, the fraction of nonthermal $\mathrm{D}^{3} \mathrm{He}$ protons $\eta_{p}^{\prime}=n_{p}^{\prime} / n_{p}$ is given by the dashed curve. 
Now we consider the effective temperature of nonthermal neutrons $T_{n}^{\prime}$. This temperature is a measure of the average kinetic energy of these neutrons $\left\langle E_{n}\right\rangle$, so it can reasonably be evaluated as

$$
T_{n}^{\prime}=\frac{2}{3}\left\langle E_{n}\right\rangle=\frac{2}{3 n_{n}^{\prime}} \int_{E_{1}}^{E_{2}} E_{n} f_{n}^{\text {tail }}\left(E_{n}\right) d E_{n} .
$$

In Fig. 10, the solid curve shows the calculated value of $T_{n}^{\prime}$ versus the thermal particle (i.e., Universe) temperature $T$. As seen, $T_{n}^{\prime}$ reaches a high level of several $\mathrm{MeV}$ and exceeds $T$ by a factor ranging within 70-300. It is possible to find constraints on the effective temperature $T_{n}^{\prime}$ using the following simple consideration. The temperature of monochromatic 14.07-MeV DT neutrons, i.e., neutrons which do not undergo collisions with plasma particles, evaluated by Eq. (42) is $T_{1}=9.4 \mathrm{MeV}$. This case apparently is nonrealistic, but it reflects the upper temperature limit. When slowing down in the plasma, the average fractional energy loss $\xi_{E_{n}}$ of a neutron per elastic scattering collision (with a proton and/or an $\alpha$ particle) is estimated to be $0.2-0.4$ in the $\mathrm{MeV}$ energy range. Only a few collisions for the neutron is enough to become thermalized. Choosing the "central" value for $\xi_{E_{n}}=0.3$, we find that the neutron loses most of the energy $\sim 90 \%$ after 3 collisions. As a result of these collisions, the monochromatic neutron temperature decreases from $T_{1}$ down to $T_{2}=0.8 \mathrm{MeV}$ that approximately reflects the lower limit. As seen, the realistic value of $T_{n}^{\prime}$ indeed lies in the $T_{1}-T_{2}$ corridor.

For comparison, the effective temperature of nonthermal $\mathrm{D}^{3} \mathrm{He}$ protons $T_{p}^{\prime}$ is shown in Fig. 10 by the dashed curve. This temperature is essentially suppressed as the protons rapidly cool down due to interaction with thermal $e^{ \pm}$(see details in [31]). It is worthwhile to remind one that $T_{p}^{\prime}$ is the most high temperature of reaction-produced charged particles $\left(p, t,{ }^{3} \mathrm{He}, \alpha\right)$. This means that $T_{n}^{\prime}$ essentially exceeds the temperature of any nonthermal as well as thermal plasma species. In view of this, an interesting question

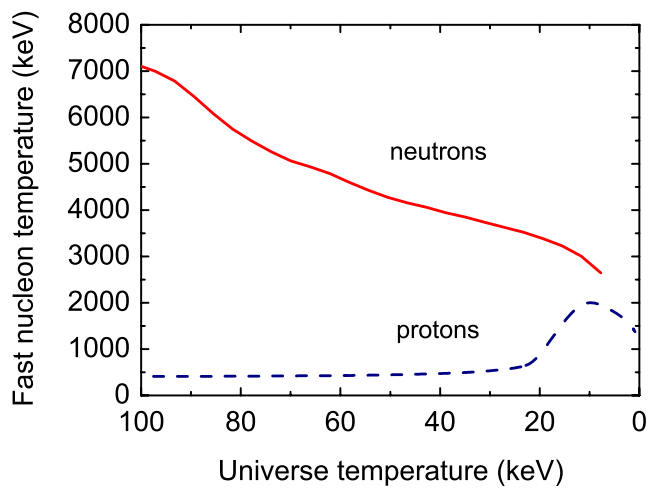

FIG. 10 (color online). The effective temperature of nonthermal neutrons $T_{n}^{\prime}$ versus the Universe temperature is shown by the solid curve. For comparison, the dashed curve gives the effective temperature of nonthermal $\mathrm{D}^{3} \mathrm{He}$ protons $T_{p}^{\prime}$. arises of whether or not the nonthermal neutron group could change the rates of individual $n$-induced reactions.

\section{Nonthermal impact on reaction rates}

First, we recall some definitions and expressions useful for evaluations of reaction rates. The rate $R_{i j}$ of nuclear reaction between plasma particles $i$ and $j$ is defined as

$$
R_{i j}=\frac{1}{1+\delta_{i j}} n_{i} n_{j}\langle\sigma v\rangle_{i j}
$$

where $\delta_{i j}$ is the Kronecker's delta, $n_{i}$ and $n_{j}$ are the number densities of reacting nuclei, and $\langle\sigma v\rangle_{i j}$ is the $i+j$ reaction rate parameter (so called the $i+j$ reactivity). For the nonrelativistic $i+j$ process, the rate parameter is given by

$$
\langle\sigma v\rangle_{i j}=\frac{1}{n_{i} n_{j}} \int f_{i}\left(\mathbf{v}_{i}\right) f_{j}\left(\mathbf{v}_{j}\right) \sigma_{i j}\left(\left|\mathbf{v}_{i}-\mathbf{v}_{j}\right|\right)\left|\mathbf{v}_{i}-\mathbf{v}_{j}\right| d \mathbf{v}_{i} d \mathbf{v}_{j},
$$

where $\sigma_{i j}$ is the $i+j$ reaction cross section, and $f_{k}(k=$ $i, j)$ is the velocity distribution function of particles $k$ having velocity $\mathbf{v}_{k}$ in the laboratory frame. The function $f_{k}$ is normalized to the particle number density $n_{k}$. After some algebra, Eq. (44) can be reduced to an expression convenient for numerical integration

$$
\begin{aligned}
\langle\sigma v\rangle_{i j}= & \frac{8 \pi^{2}}{n_{i} n_{j}} \int_{0}^{\infty} v_{i} f_{i}\left(v_{i}\right) \int_{0}^{\infty} v_{j} f_{j}\left(v_{j}\right) \\
& \times \int_{\left|v_{i}-v_{j}\right|}^{v_{i}+v_{j}} v^{2} \sigma(v) d v d v_{i} d v_{j},
\end{aligned}
$$

where $v_{k}=\left|\mathbf{v}_{k}\right|$ and $v$ is the relative speed $\left|\mathbf{v}_{i}-\mathbf{v}_{j}\right|$. If particles $i$ and $j$ have Maxwellian distribution functions $f_{i, \mathrm{M}}$ and $f_{i, \mathrm{M}}$ with temperatures $T_{i}$ and $T_{j}$, respectively, the $i+j$ reactivity takes the following densityindependent form

$$
\begin{aligned}
\langle\sigma v\rangle_{i j, \mathrm{M}} & =\left(\frac{8}{\pi m_{r}}\right)^{1 / 2} T_{\mathrm{av}}^{-3 / 2} \int_{0}^{\infty} E \sigma(E) \exp \left(-\frac{E}{T_{\mathrm{av}}}\right) d E, \\
T_{\mathrm{av}} & =\frac{m_{j}}{m_{i}+m_{j}} T_{i}+\frac{m_{i}}{m_{i}+m_{j}} T_{j} .
\end{aligned}
$$

In these equations, $m_{r}$ is the reduced mass of these particles, $E$ is their kinetic energy in the center-of-mass frame, and $T_{\mathrm{av}}$ is some average Maxwellian temperature. If both distributions have the same temperature $T$, then $T_{\mathrm{av}}=T$ and Eq. (46a) gives the classical formula for reactivity in a Maxwellian plasma.

Equations (46) make it possible to simplify a description of nonthermal reactions by using a two-temperature Maxwellian (2TM) model for particle distribution functions [42]. In this model, the realistic distribution function of particles $k$ is approximated by a superposition of two Maxwellian functions 


$$
f_{k}\left(\mathbf{v}_{k} ; \mathbf{v}_{k}^{\prime}\right) \simeq f_{k, \mathrm{M}}\left(\mathbf{v}_{k}\right)+f_{k, \mathrm{M}}^{\prime}\left(\mathbf{v}_{k}^{\prime}\right) .
$$

Here $f_{k, \mathrm{M}}$ describes the behavior of bulk thermal particles with density $n_{k \text {,bulk }}$ and temperature $T_{k \text {, bulk }}\left(\equiv T_{k}\right)$, while the function $f_{k, \mathrm{M}}^{\prime}$ is introduced to model the ensemble of nonthermal particles with some density $n_{k}^{\prime}$ and temperature $T_{k}^{\prime}>T_{k}$. Apparently, the total number density $n_{k}=$ $n_{k \text {,bulk }}+n_{k}^{\prime}$. Substituting Eq. (47) into Eq. (44) and taking into account Eqs. (46), we can rewrite the general expression for $\langle\sigma v\rangle_{i j}$ as

$$
\begin{aligned}
\langle\sigma v\rangle_{i j} \simeq & \eta_{i} \eta_{j}\langle\sigma v\rangle_{i j, \mathrm{M}}^{b b}+\eta_{i}^{\prime} \eta_{j}\langle\sigma v\rangle_{i j, \mathrm{M}}^{f b}+\eta_{i} \eta_{j}^{\prime}\langle\sigma v\rangle_{i j, \mathrm{M}}^{b f} \\
& +\eta_{i}^{\prime} \eta_{j}^{\prime}\langle\sigma v\rangle_{i j, \mathrm{M}}^{f f}
\end{aligned}
$$

where $\eta_{k}=n_{k \text {,bulk }} / n_{k}$ and $\eta_{k}^{\prime}=n_{k}^{\prime} / n_{k}$ are the fractions of bulk and nonthermal particles $k(=i, j)$ in the total plasma $k$ component, respectively. This formula shows the partial contributions to $\langle\sigma v\rangle_{i j}$ resulting from bulk-bulk, fast-bulk, and fast-fast particle interactions. The subscript at each term (reactivity) on the right-hand side of Eq. (48) denotes the sort of reacting particles as bulk $(b)$ or fast $(f)$. All these reactivities are determined by Eqs. (46).

Now we can examine how the non-Maxwellian tail of the realistic neutron distribution affects the rate parameters of individual $n$-induced reactions. In this connection, note that there is a difference between mechanisms of nonthermal impact on exoergic reactions induced by charged particles and neutrons. The cross sections of charged particle reactions usually increase with increasing energy, so fast particles can enhance the reaction rates. Contrariwise, the cross sections of exoergic neutronic processes commonly decrease with increasing neutron energy. These processes therefore are weakly sensitive to fast neutrons unless strong resonances appear in a high-energy region. At the same time however an increase of fast neutron population leads to a redistribution of the whole neutronic component, so that the amount of thermal neutrons-for which the reaction cross sections are large-somewhat decreases. Thus, such redistribution can cause a weakening of the respective rates of exoergic reactions. This specific effect however is unlikely to manifest in the BBN epoch because the evaluated fast neutron fraction $\eta_{n}^{\prime}$ proves to be rather small (see Fig. 9).

The most promising candidates to observe a clear signature of nonthermal neutron effects are endoergic (i.e., threshold) neutronic reactions. These reactions cannot proceed at energies below their thresholds $E_{\text {thr }}$ and should be strongly influenced by fast neutrons. Such processes having negative $Q$ values-for example, $n$-induced breakup as well as various reverse reactions-widely occur in the $\mathrm{BBN}$ epoch. In the present work, we examine the influence of nonthermal neutrons on breakups of loosely bound D, ${ }^{7} \mathrm{Li}$, and ${ }^{7} \mathrm{Be}$ nuclei synthesized in the primordial plasma. Such a choice of processes is partly motivated by recent speculations [31] showing that under certain conditions these disintegration reactions may in principle affect the abundances of primordial D and ${ }^{7} \mathrm{Li}$. Thus, we consider the following two-body breakup reactions induced by neutrons:

$$
\begin{array}{cc}
n+\mathrm{D} \rightarrow n+n+p, & Q=-2.22 \mathrm{MeV}, \\
n+{ }^{7} \mathrm{Li} \rightarrow n+t+\alpha, & Q=-2.47 \mathrm{MeV}, \\
n+{ }^{7} \mathrm{Be} \rightarrow n+{ }^{3} \mathrm{He}+\alpha, & Q=-1.59 \mathrm{MeV} .
\end{array}
$$

The $n+\mathrm{D}$ and $n+{ }^{7} \mathrm{Li}$ reaction cross sections taken from ENDF [35] and JENDL [43], respectively, are shown in Fig. 11 by the solid curves. The $n+{ }^{7} \mathrm{Be}$ reaction cross section is unavailable in the literature and not plotted here. However, simple arguments [16] indicate that this cross section can be assumed to be close to that for the $n+{ }^{7} \mathrm{Li}$ reaction. Although such assumption suffers from some inaccuracy at energies close to threshold $E_{\mathrm{thr}}$, this is not dramatic for our study as the near-threshold region does not contribute much to the $n+{ }^{7} \mathrm{Be}$ reaction rate. The dotted curve in Fig. 11 shows the realistic neutron distribution function calculated at $T_{9}=0.9$. As seen, the nonMaxwellian distribution tail overlaps the energy range where the considered reactions have sizable cross sections. So we may expect that the nonthermal neutrons populating this tail can significantly enhance the $n+X$ breakup reactions $\left(X=\mathrm{D},{ }^{7} \mathrm{Li},{ }^{7} \mathrm{Be}\right)$.

This conjecture is well confirmed in Fig. 12 demonstrating the astrophysical rate coefficients for these reactions $N_{\mathrm{A}}\langle\sigma v\rangle_{n X}$, where $N_{\mathrm{A}}$ is the Avogadro's number. The solid curves show $N_{\mathrm{A}}\langle\sigma v\rangle_{n X}$ obtained for the realistic neutron energy distributions using Eq. (45). These realistic rate coefficients are compared with the Maxwellian ones $N_{\mathrm{A}}\langle\sigma v\rangle_{n X, \mathrm{M}}$ given in Fig. 12 by the dashed curves. It is clearly seen that the nonthermal neutrons strongly support the breakup reactions as the Universe cools down. Furthermore, the Maxwellian-based description of the

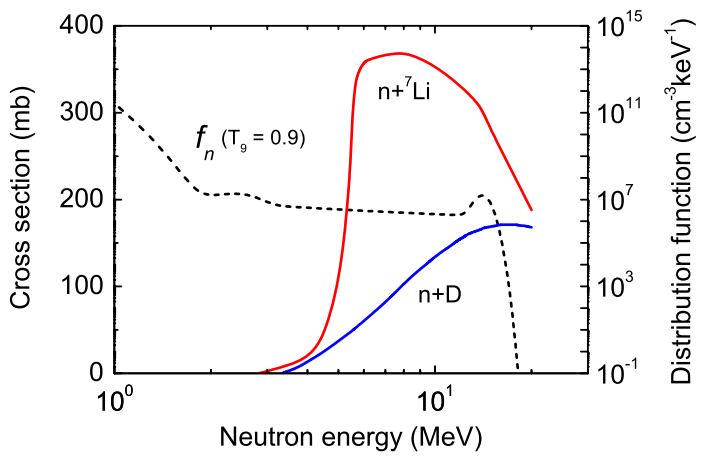

FIG. 11 (color online). The solid curves show the cross sections of the $n+\mathrm{D} \rightarrow n+n+p$ and $n+{ }^{7} \mathrm{Li} \rightarrow n+t+\alpha$ breakup reactions as a function of incident neutron energy. The dotted curve presents the realistic neutron distribution function at the Universe temperature $T_{9}=0.9$. 




FIG. 12 (color online). The astrophysical rate coefficients for the $n+X$ breakup reactions $\left(X=\mathrm{D},{ }^{7} \mathrm{Li},{ }^{7} \mathrm{Be}\right)$ as a function of the Universe temperature. Shown are the realistic rate coefficients $N_{\mathrm{A}}\langle\sigma v\rangle_{n X}$, the Maxwellian ones $N_{\mathrm{A}}\langle\sigma v\rangle_{n X, \mathrm{M}}$, and the rate coefficient $N_{\mathrm{A}}\langle\sigma v\rangle_{n X, 2 \mathrm{TM}}$ estimated within the 2TM model.

breakup reactivities proves to be fully useless at temperatures $T_{9}$ below some critical value $T_{\mathrm{cr}} \simeq 1.2$.

An interesting question is to what degree of accuracy the 2TM model could describe these reactions. In Fig. 13, we approximate the realistic neutron distribution obtained at $T_{9}=0.7$ by two Maxwellian functions presenting the ensembles of bulk and fast neutrons with parameters $\left(\eta_{n}, T_{n}\right)$ and $\left(\eta_{n}^{\prime}, T_{n}^{\prime}\right)$, respectively. Here $\eta_{n}=1-\eta_{n}^{\prime}$, $T_{n}=T_{9}$ (Universe temperature), while $\eta_{n}^{\prime}$ and $T_{n}^{\prime}$ are plotted in Figs. 9 and 10. Figure 13 shows that the Maxwellian function cannot accurately reproduce the realistic distribution tail truncated at some energy close to the DT neutron birth energy. However, this local discrepancy may not dramatically affect some averaged quantities such as $\langle\sigma v\rangle$. Assuming the target nuclei $X=\mathrm{D},{ }^{7} \mathrm{Li},{ }^{7} \mathrm{Be}$ purely bulk $\left(\eta_{X}=1\right)$, one can easily reduce Eq. (48) to

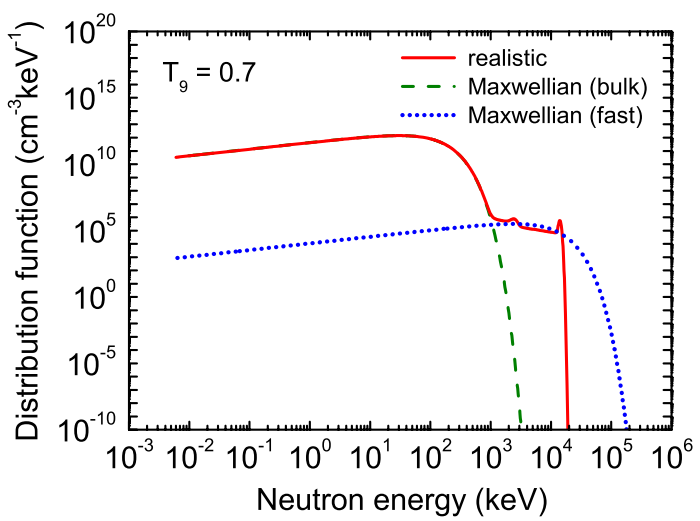

FIG. 13 (color online). The approximation of the realistic neutron distribution function (shown by the solid curve) by two Maxwellian distributions. The dashed and dotted curves describe the ensembles of bulk and fast neutrons, respectively.

$$
\begin{aligned}
\langle\sigma v\rangle_{n X} & \simeq\langle\sigma v\rangle_{n X, 2 \mathrm{TM}} \\
& =\left(1-\eta_{n}^{\prime}\right)\langle\sigma v\rangle_{n X, \mathrm{M}}^{b b}+\eta_{n}^{\prime}\langle\sigma v\rangle_{n X, \mathrm{M}}^{f b} .
\end{aligned}
$$

The rate coefficients $N_{\mathrm{A}}\langle\sigma v\rangle_{n X, 2 \mathrm{TM}}$ are shown in Fig. 12 by open circles. We see that the $2 \mathrm{TM}$ model provides a remarkable description of the realistic rate coefficients.

\section{CONCLUSIONS}

In the present work, we have presented the first analysis of the realistic neutron component in the early Universe plasma during the BBN epoch. The respective energy distribution function of plasma neutrons has been calculated within the appropriate plasma-kinetic model. The main characteristics of this neutron component have been examined in detail. Briefly summarizing the results of our study, we conclude the following:

(1) Fast neutrons generated in the $\mathrm{T}(d, n)^{4} \mathrm{He}$ and $\mathrm{D}(d, n)^{3} \mathrm{He}$ reactions in the plasma cause the perturbation of the neutron distribution function, so that its high-energy tail is essentially enhanced and departs from a conventional Maxwellian function. The competing source of nonthermal neutron productiondue to elastic collisions of bulk neutrons with fast charged products of nuclear reactions-has been found to be not significant. It becomes important to the production of neutrons with energies only less than $1 \mathrm{MeV}$.

(2) The fraction of nonthermal neutrons $\eta_{n}^{\prime}$ in the total neutron component in the plasma is at a level of $10^{-2} \%-10^{-3} \%$. At the same time, their effective temperature $T_{n}^{\prime}$ reaches several $\mathrm{MeV}$ and exceeds the Universe temperature in the BBN epoch by about 2 orders of magnitude. These neutron parameters have record values; they are much higher than the respective parameters $\left(\eta_{c}^{\prime}, T_{c}^{\prime}\right)$ for nonthermal charged particles $c\left(=p, t,{ }^{3} \mathrm{He}, \alpha\right)$ in the plasma. 
(3) The nonthermal neutron influence on some individual neutronic processes has been examined. On the example of the $n+X$ breakup reactions $(X=\mathrm{D}$, ${ }^{7} \mathrm{Li},{ }^{7} \mathrm{Be}$ ), we have demonstrated that the nonthermal neutrons can strongly enhance the rates of endoergic processes. Furthermore, there is a critical plasma temperature $T_{\mathrm{cr}} \simeq 1.2$ below which the discrepancy between the realistic rate coefficients $N_{\mathrm{A}}\langle\sigma v\rangle_{n X}$ and those obtained within the Maxwellian approximation $N_{\mathrm{A}}\langle\sigma v\rangle_{n X, \mathrm{M}}$ is so appreciable that the Maxwellian-based description of the breakup reactions becomes fully useless.

(4) It has been shown that the 2TM model can serve as a convenient tool for a proper description of reactions involving non-Maxwellian particles, provided the number density and effective temperature of these particles are determined properly. This result is of particular methodical significance.

An important question still remains-whether or not the nonthermal neutrons could affect reaction kinetics in the early Universe and change the predictions of standard BBN. A number of nonthermal forward as well as reverse reactions should be incorporated in the SBBN network. As for the nonthermal $X$ breakups examined in the paper, their rates $R_{n X \text {, nonth }}$ prove to be lower than the thermal rates $R_{i X, \text { th }}$ for major competing reactions of $X$ depletion: $\mathrm{D}(d, n){ }^{3} \mathrm{He}, \mathrm{D}(d, p) \mathrm{T},{ }^{7} \mathrm{Li}(p, \alpha){ }^{4} \mathrm{He}$, and $\left.{ }^{7} \mathrm{Be}(n, p)\right)^{7} \mathrm{Li}$. It is found that for each target nucleus $X$ the ratio $R_{n X, \text { nonth }} / R_{i X, \text { th }} \sim 10^{-3}$ at maximum. At the same time, however, nonthermal neutrons can appreciably enhance some other reactions. A process of particular interest is $d+d \rightleftarrows n+{ }^{3} \mathrm{He}$, for which the reverse ${ }^{3} \mathrm{He}(n, d)$ reactivity can become almost comparable with the forward $d(d, n)$ reactivity [32]. This may somewhat prevent D depletion and thereby cause an additional emission of neutrons predominantly via the resonant $\mathrm{T}(d, n)$ reaction. The strength of this emission has not been examined yet. In this regard, it is worth noting that an injection of neutrons at a level of $n / p \sim 10^{-5}$ was found to cause significant ${ }^{7} \mathrm{Be}$ destruction [44]. We address the study of SBBN predictions allowing for nonthermal reactions in a future publication.

\section{ACKNOWLEDGMENTS}

The authors thank Dr. Makoto Nakamura for his help in the analysis of neutron energy loss due to nuclear scattering processes.
[1] R. V. Wagoner, W. A. Fowler, and F. Hoyle, Astrophys. J. 148, 3 (1967).

[2] R. V. Wagoner, Astrophys. J. Suppl. Ser. 18, 247 (1969).

[3] D. N. Spergel et al., Astrophys. J. Suppl. Ser. 148, 175 (2003).

[4] D. N. Spergel et al., Astrophys. J. Suppl. Ser. 170, 377 (2007).

[5] K. Jedamzik and M. Pospelov, New J. Phys. 11, 105028 (2009).

[6] R. H. Cyburt, B. D. Fields, and K. A. Olive, J. Cosmol. Astropart. Phys. 11 (2008) 012.

[7] R. H. Cyburt, J. Ellis, B. D. Fields, K. A. Olive, and V. C. Spanos, J. Cosmol. Astropart. Phys. 11 (2006) 014.

[8] M. Kusakabe, T. Kajino, R. N. Boyd, T. Yoshida, and G. T. Mathews, Astrophys. J. 680, 846 (2008).

[9] D. Cumberbatch, K. Ichikawa, M. Kawasaki, and K. Kohri, Phys. Rev. D 76, 123005 (2007).

[10] K. Kohri and F. Takayama, Phys. Rev. D 76, 063507 (2007).

[11] C. Bird, K. Koopmans, and M. Pospelov, Phys. Rev. D 78, 083010 (2008).

[12] R. H. Cyburt, J. Ellis, B. D. Fields, F. Luo, K. A. Olive, and V. C. Spanos, J. Cosmol. Astropart. Phys. 10 (2010) 032.

[13] M. Pospelov and J. Pradler, Annu. Rev. Nucl. Part. Sci. 60, 539 (2010).

[14] M. Kawasaki and M. Kusakabe, Phys. Rev. D 83, 055011 (2011).

[15] V. T. Voronchev, Y. Nakao, and M. Nakamura, J. Cosmol. Astropart. Phys. 05 (2008) 010.
[16] V. T. Voronchev, N. Nakamura, and Y. Nakao, J. Cosmol. Astropart. Phys. 05 (2009) 001.

[17] R. N. Boyd, C. R. Brune, G. M. Fuller, and C. J. Smith, Phys. Rev. D 82, 105005 (2010).

[18] R. V. Wagoner, Astrophys. J. 179, 343 (1973).

[19] L. Kawano, Report No. FERMILAB-PUB-92/04-A, 1992.

[20] R. H. Cyburt and M. Pospelov, arXiv:0906.4373.

[21] N. Chakraborty, B. D. Fields, and K. A. Olive, Phys. Rev. D 83, 063006 (2011).

[22] C. J. Smith, G. M. Fuller, and M. S. Smith, Phys. Rev. D 79, 105001 (2009).

[23] G. M. Fuller and C. J. Smith, Phys. Rev. D 82, 125017 (2010).

[24] B. Wang, C. A. Bertulani, and A. B. Balantekin, Phys. Rev. C 83, 018801 (2011).

[25] NACRE Collaboration, http://pntpm.ulb.ac.be/Nacre/.

[26] K. M. Nollett and S. Burles, Phys. Rev. D 61, 123505 (2000).

[27] R. H. Cyburt, Phys. Rev. D 70, 023505 (2004).

[28] P. Descouvemont, A. Adahchour, C. Angulo, A. Coc, and E. Vangioni-Flam, At. Data Nucl. Data Tables 88, 203 (2004).

[29] P. D. Serpico, S. Esposito, F. Iocco, G. Mangano, G. Miele, and O. Pisanti, J. Cosmol. Astropart. Phys. 12 (2004) 010.

[30] C. Iliadis, R. Longland, A. E. Champagne, A. Coc, and R. Fitzgerald, Nucl. Phys. A841, 31 (2010).

[31] V. T. Voronchev, Y. Nakao, and M. Nakamura, Astrophys. J. 725, 242 (2010). 
[32] V. T. Voronchev, Y. Nakao, and M. Nakamura, J. Phys. G 38, 015201 (2011).

[33] M. Kawasaki, K. Kohri, and T. Moroi, Phys. Rev. D 71, 083502 (2005).

[34] R. J. Gould, Astrophys. J. 417, 12 (1993).

[35] Evaluated Nuclear Data File (ENDF), http:// www.nndc.bnl.gov/exfor/endf00.jsp.

[36] F. Ajzenberg-Selove, Nucl. Phys. A490, 1 (1988).

[37] For example, F. Reif, Fundamentals of Statistical and Thermal Physics (McGraw-Hill, New York, 1965).

[38] H. Brysk, Plasma Phys. 15, 611 (1973).
[39] M. Nakamura, Y. Nakao, V. T. Voronchev, K. Hegi, H. Nakamura, and O. Mitarai, J. Phys. Soc. Jpn. 75, 024801 (2006).

[40] For example, G. I. Bell and S. Glasstone, Nuclear Reactor Theory (Van Nostrand Reinhold Co., New York, 1970).

[41] K. H. Beckurts and K. Wirts, Neutron Physics (SpringerVerlag, New York, 1964).

[42] M. Nakamura, V. T. Voronchev, and Y. Nakao, Phys. Lett. A 359, 663 (2006).

[43] Japanese Evaluated Nuclear Data Library (JENDL), http:// wwwndc.jaea.go.jp/jendl/j40/j40.html.

[44] K. Jedamzik, Phys. Rev. D 70, 063524 (2004). 